\title{
On Complete Convergence and Strong Law for Weighted Sums of i.i.d. Random Variables
}

\author{
Pingyan Chen, ${ }^{1}$ Xiaofang $\mathrm{Ma}^{2}{ }^{2}$ and Soo Hak Sung ${ }^{3}$ \\ ${ }^{1}$ Department of Mathematics, Jinan University, Guangzhou 510630, China \\ ${ }^{2}$ Department of Statistics, Jinan University, Guangzhou 510630, China \\ ${ }^{3}$ Department of Applied Mathematics, Pai Chai University, Taejon 302-735, Republic of Korea
}

Correspondence should be addressed to Soo Hak Sung; sungsh@pcu.ac.kr

Received 20 April 2014; Accepted 11 June 2014; Published 25 June 2014

Academic Editor: Simeon Reich

Copyright (C) 2014 Pingyan Chen et al. This is an open access article distributed under the Creative Commons Attribution License, which permits unrestricted use, distribution, and reproduction in any medium, provided the original work is properly cited.

We improve and generalize the result of Stout (1974, Theorem 4.1.3). In particular, the sharp moment conditions are obtained and some well-known results can be obtained as special cases of the main result. The method of the proof is completely different from that in Stout. We also improve and generalize Li et al. (1995) strong law for weighted sums of i.i.d. random variables.

\section{Introduction and the Main Result}

Stout [1] obtained the following celebrated result.

Theorem A (see Theorem 4.1.3 in [1] or p. 1556 in Stout [2]). Let $\left\{X, X_{n} \geq 1\right\}$ be a sequence of independent and identically distributed random variables with $E X=0$ and $E|X|^{2 / \alpha}<\infty$ for some $0<\alpha \leq 1$. Suppose that $\left\{a_{n k}, 1 \leq k \leq n, n \geq 1\right\}$ is a sequence of constants with

$$
\max _{1 \leq k \leq n}\left|a_{n k}\right| \leq K n^{-\alpha}
$$

for some constant $0<K<\infty$ and

$$
\lim _{n \rightarrow \infty} \log n \sum_{k=1}^{n} a_{n k}^{2}=0 .
$$

Then

$$
\sum_{n=1}^{\infty} P\left\{\left|\sum_{k=1}^{n} a_{n k} X_{k}\right|>\varepsilon\right\}<\infty, \quad \forall \varepsilon>0 .
$$

Formula (3) is called complete convergence and this concept was introduced by Hsu and Robbins [3]. Sung [4] and Cheng and Wang [5] extended Theorem A to random elements taking values in a Banach space. Wu [6] and Sung [7] extended Theorem A to negatively dependent random variables. It should be pointed out that they all used some exponential inequalities to prove their result and hence the proofs are similar to that of Theorem A except for more computational complexity.

When $1 / 2<\alpha \leq 1$, (1) clearly implies (2). Set $a_{n k}=n^{-\alpha}$ for $1 \leq k \leq n$ and $n \geq 1$. Then (3) reduces to

$$
\sum_{n=1}^{\infty} P\left\{\left|\sum_{k=1}^{n} X_{k}\right|>\varepsilon n^{\alpha}\right\}<\infty, \quad \forall \varepsilon>0
$$

which is equivalent to $E|X|^{2 / \alpha}<\infty$ and $E X=0$ by Katz [8] and Baum and Katz [9]. Hence, the moment conditions of Theorem $\mathrm{A}$ are sharp when $1 / 2<\alpha \leq 1$.

Next, we consider the case of $0<\alpha \leq 1 / 2$. Lai [10] showed that

$$
\sum_{n=1}^{\infty} P\left\{\left|\sum_{k=1}^{n} X_{k}\right|>\varepsilon \sqrt{n \log n}\right\}<\infty
$$

for $\varepsilon>\sqrt{2}$ provided $E X=0, E X^{2}=1$, and $E X^{4} / \log ^{2}|X|<$ $\infty$. Hence, for any $\varepsilon>0$,

$$
\sum_{n=1}^{\infty} P\left\{\left|\sum_{k=1}^{n} X_{k}\right|>\varepsilon c_{n} \sqrt{n \log n}\right\}<\infty
$$


if $c_{n} \rightarrow \infty$. Set $a_{n k}=\left(c_{n} \sqrt{n \log n}\right)^{-1}$ for $1 \leq k \leq n$ and $n \geq 1$. Then (1) and (2) hold for $\alpha=1 / 2$. Note that the moment conditions are weaker than those of Theorem A when $\alpha=$ $1 / 2$. Hence, the moment conditions of Theorem A may not be optimal for a special case of weighted sums. But it is not known whether the moment conditions of Theorem $A$ are not optimal when $0<\alpha \leq 1 / 2$.

In this paper, we will discuss the optimized moment conditions of Theorem A when $0<\alpha \leq 1 / 2$. We obtain a more generalized complete convergence result for weighted sums which includes the result of Lai [10]. Our method used is completely different from those in Lai [10] and Stout [1].

Li et al. [11] obtained the following celebrated result.

Theorem B (see Theorem 3.1 in [11]). Let $\left\{X, X_{n} \geq 1\right\}$ be a sequence of independent and identically distributed random variables with $E X=0$ and $E|X|^{1 / \alpha}<\infty$ for some $0<\alpha \leq 1$. Suppose that $\left\{a_{n k}, 1 \leq k \leq n, n \geq 1\right\}$ is a sequence of constants with (1) for some constant $0<K<\infty$ and

$$
\lim _{n \rightarrow \infty} n^{\gamma} \sum_{k=1}^{n} a_{n k}^{2}=0
$$

where $\gamma>2 \alpha-1$ if $1 / 2 \leq \alpha \leq 1$ and $\gamma>0$ if $0<\alpha<1 / 2$. Then

$$
\lim _{n \rightarrow \infty} \sum_{k=1}^{n} a_{n k} X_{k}=0 \text { a.s. }
$$

Theorem $\mathrm{B}$ has been extended and improved by many authors. Jing and Liang [12] extended and improved to negatively associated (NA) random variables, Budsaba et al. [13] to certain types of $U$-statistics bases on this kind of weighted sums of NA random variables, and Thanh and Yin [14] to the random weighted sums. In particular, under the condition

$$
\limsup _{n \rightarrow \infty} \log n \sum_{k=1}^{n} a_{n k}^{2}<\infty,
$$

Jing and Liang [12] showed that

$$
\limsup _{n \rightarrow \infty}\left|\sum_{k=1}^{n} a_{n k} X_{k}\right|<\infty \text { a.s. }
$$

when $0<\alpha<1 / 2$.

Is it possible to find the sharp bound of (10)? In this paper, we will give a definite answer to the question under more general case.

Throughout this paper, $c$ represents a positive constant which may vary in different places, $[x]$ denotes the integer part of $x$, and $f(x) \sim g(x)$ means $f(x) / g(x) \rightarrow 1$ as $x \rightarrow \infty$. It proves convenient to define $\log x=\max \{1, \ln x\}$, where $\ln x$ denotes the natural logarithm.

Now, we are ready to state the main results, and the proofs will be given in the next section.

Theorem 1. Let $r>0,0<\alpha \leq 1 / 2, g(x)$ be an increasing and regular varying function at infinity with index $\alpha$ and let $h(x)$ be the inverse function of $g(x)$. Let $\left\{X, X_{n} \geq 1\right\}$ be a sequence of independent and identically distributed random variables with $E X=0, E X^{2}=1$, and $E h^{r}(|X|)|X|^{2} / \log h(|X|)<\infty$. Suppose that $\left\{a_{n k}, 1 \leq k \leq n, n \geq 1\right\}$ is a sequence of constants such that

$$
\max _{1 \leq k \leq n}\left|a_{n k}\right| \leq K^{-1}(n)
$$

for some constant $K>0$ and

$$
\lim _{n \rightarrow \infty} \log n \sum_{k=1}^{n} a_{n k}^{2}=\rho
$$

for some constant $0 \leq \rho<\infty$. Then

$$
\sum_{n=1}^{\infty} n^{r-1} P\left\{\left|\sum_{k=1}^{n} a_{n k} X_{k}\right|>\varepsilon\right\} \begin{cases}<\infty & \text { if } \varepsilon>\sqrt{2 r \rho}, \\ =\infty & \text { if } 0<\varepsilon<\sqrt{2 r \rho} .\end{cases}
$$

Remark 2. Recall that a measurable function $f(x)$ is said to be regularly varying at infinity with index $\beta$ if it is positive on $[0, \infty)$ and

$$
\lim _{x \rightarrow \infty} \frac{f(\lambda x)}{f(x)}=\lambda^{\beta}, \quad \forall \lambda>0 .
$$

We refer to Bingham et al. [15] for other equivalent definitions and for detailed and comprehensive study of properties of regularly varying functions. For example, if $\beta>-1$,

$$
\sum_{k=1}^{n} f(k) \leq c_{\beta} n f(n)
$$

and if $\beta<-1$,

$$
\sum_{k=n}^{\infty} f(k) \leq C_{\beta} n f(n),
$$

where $c_{\beta}>0$ and $C_{\beta}>0$ are constants depending only on $\beta$.

Remark 3. When $r=1$ and $g(x)=x^{\alpha}$, the last moment condition of Theorem 1 is reduced to $E|X|^{1 / \alpha+2} / \log |X|<\infty$. When $0<\alpha \leq 1 / 2,1 / \alpha+2 \leq 2 / \alpha$ and so the moment conditions in Theorem 1 are strictly weaker than those in Theorem A.

Remark 4. Let $a_{n k}=g^{-1}(n)$ if $1 \leq k \leq\left[g^{2}(n) / \log n\right]$ and $a_{n k}=0$ if $k>\left[g^{2}(n) / \log n\right]$. Then, it is easy to show that

$$
\lim _{n \rightarrow \infty} \log n \sum_{k=1}^{n}\left|a_{n k}\right|^{2}=1 \text {. }
$$

Hence, (11) and (12) hold. Under the moment conditions of Theorem 1

$$
\sum_{n=1}^{\infty} n^{r-1} P\left\{\left|\sum_{k=1}^{\left[g^{2}(n) / \log n\right]} X_{k}\right|>\varepsilon g(n)\right\}<\infty
$$

for all $\varepsilon>\sqrt{2 r}$. On the other hand, it is easy to show that if the above formula holds for some $\varepsilon>0$, then $E h^{r}(|X|)|X|^{2} / \log h(|X|)<\infty$ by the similar argument as in Lai [10]. Thus, the moment conditions of Theorem 1 are sharp in the sense that the moment conditions on $X$ cannot be weakened. 
Remark 5. Let $g(x)=\sqrt{x \log x}$ and $a_{n k}=g^{-1}(n)$ for $1 \leq$ $k \leq n$ and $n \geq 1$. Then, by Theorem 1 , the moment conditions $E X=0, E X^{2}=1$, and $E\left(X^{2} / \log |X|\right)^{r+1}<\infty$ imply that

$$
\sum_{n=1}^{\infty} n^{r-1} P\left\{\left|\sum_{k=1}^{n} X_{k}\right|>\varepsilon \sqrt{n \log n}\right\} \begin{cases}<\infty & \text { if } \varepsilon>\sqrt{2 r}, \\ =\infty & \text { if } 0<\varepsilon<\sqrt{2 r} .\end{cases}
$$

So, the sufficient part of Theorem 3 in Lai [10] is a special case of Theorem 1.

By Theorem 1 and Borel-Cantelli lemma, we have the following corollary.

Corollary 6. Under the conditions of Theorem 1, let $r=1$, $\left\{X_{n k}, 1 \leq k \leq n, n \geq 1\right\}$ be an array of independent random variables with the same distribution as $X$. Then

$$
\limsup _{n \rightarrow \infty}\left|\sum_{k=1}^{n} a_{n k} X_{n k}\right|=\sqrt{2 \rho} \text { a.s. }
$$

In particular, the moment conditions $E X=0, E X^{2}=1$, and $E X^{4} / \log ^{2}|X|<\infty$ imply that

$$
\limsup _{n \rightarrow \infty} \frac{\left|\sum_{k=1}^{n} X_{n k}\right|}{\sqrt{2 n \log n}}=1 \text { a.s. }
$$

Remark 7. Formula (21) is called the law of single logarithm which is due to Hu and Weber [16]. They proved it under the strong moment condition $E X^{4}<\infty$. Qi [17] and Li et al. [18] independently proved that (21) is equivalent to conditions $E X=0, E X^{2}=1$, and $E X^{4} / \log ^{2}|X|<\infty$. In particular, $\mathrm{Li}$ et al. [18] gave a version of random elements taking values in a Banach space.

Theorem 8. Let $0<\alpha<1 / 2, g(x)$ be an increasing and regular varying function at infinity with index $\alpha$ and let $h(x)$ be the inverse function of $g(x)$. Let $\left\{X, X_{n} \geq 1\right\}$ be a sequence of independent and identically distributed random variables with $E X=0, E X^{2}=1$, and $E h(|X|)<\infty$. Suppose that $\left\{a_{n k}, 1 \leq k \leq n, n \geq 1\right\}$ is a sequence of constants such that (11) holds for some constant $K>0$ and

$$
\limsup _{n \rightarrow \infty} \log n \sum_{k=1}^{n} a_{n k}^{2}=\rho
$$

for some constant $0 \leq \rho<\infty$. Then

$$
\limsup _{n \rightarrow \infty}\left|\sum_{k=1}^{n} a_{n k} X_{k}\right| \leq \sqrt{2 \rho} \text { a.s. }
$$

Remark 9. For any $p \in(0,1)$, set

$$
a_{n k}=(4 n \pi)^{1 / 4}(\log n)^{-1 / 2}\left(\begin{array}{l}
n \\
k
\end{array}\right) p^{k}(1-p)^{n-k} .
$$

Then, by Embrechts and Maejima [19],

$$
\begin{gathered}
\max _{1 \leq k \leq n} a_{n k} \sim \frac{1}{\pi^{1 / 4}(p(1-p))^{1 / 2}} \cdot \frac{1}{n^{1 / 4}(\log n)^{1 / 2}}, \\
\log n \sum_{k=1}^{n} a_{n k}^{2} \sim 2^{-1}(p(1-p))^{-1 / 2}
\end{gathered}
$$

as $n \rightarrow \infty$. Hence, from Theorem $8, E X=0, E X^{2}=$ 1, $E\left(|X|^{2} / \log |X|\right)^{2}<\infty$ imply that

$$
\begin{aligned}
& \limsup _{n \rightarrow \infty}(4 n \pi)^{1 / 4}(\log n)^{-1 / 2}\left|\sum_{k=1}^{n}\left(\begin{array}{l}
n \\
k
\end{array}\right) p^{k}(1-p)^{n-k} X_{k}\right| \\
& \leq(p(1-p))^{-1 / 4} \text { a.s. }
\end{aligned}
$$

In fact, Lai [20] has proved that

$$
\begin{aligned}
& \limsup _{n \rightarrow \infty}(4 n \pi)^{1 / 4}(\log n)^{-1 / 2}\left|\sum_{k=1}^{n}\left(\begin{array}{l}
n \\
k
\end{array}\right) p^{k}(1-p)^{n-k} X_{k}\right| \\
& =(p(1-p))^{-1 / 4} \text { a.s. }
\end{aligned}
$$

if and only if $E X=0, E X^{2}=1, E\left(|X|^{2} / \log |X|\right)^{2}<\infty$. Therefore, both the upper bound and the moment condition of Theorem 8 are sharp.

Remark 10. Let $\alpha, \beta>0$ and $1 / \alpha+1 / \beta=1 / 2$. Let $\left\{b_{n k}, 1 \leq k \leq n, n \geq 1\right\}$ be a sequence of constants with $\limsup _{n \rightarrow \infty} n^{-1} \sum_{k=1}^{n}\left|b_{n k}\right|^{\alpha}<\infty$. Set $a_{n k}=(\sqrt{n \log n})^{-1} b_{n k}$. Then, it is easy to show that

$$
\max _{1 \leq k \leq n}\left|a_{n k}\right| \leq K\left(n^{1 / \beta} \sqrt{\log n}\right)^{-1}
$$

for some $K>0$ and

$$
\begin{aligned}
\rho & =\limsup _{n \rightarrow \infty} \log n \sum_{k=1}^{n}\left|a_{n k}\right|^{2}=\limsup _{n \rightarrow \infty} n^{-1} \sum_{k=1}^{n}\left|b_{n k}\right|^{2} \\
& \leq \limsup _{n \rightarrow \infty}\left(n^{-1} \sum_{k=1}^{n}\left|b_{n k}\right|^{\alpha}\right)^{2 / \alpha}<\infty
\end{aligned}
$$

by Hölder's inequality. Hence, from Theorem $8, E X=$ $0, E X^{2}=1$, and $E\left(|X|^{\beta} /(\log |X|)^{\beta / 2}\right)<\infty$ imply that

$$
\limsup _{n \rightarrow \infty} \frac{\left|\sum_{k=1}^{n} b_{n k} X_{k}\right|}{\sqrt{n \log n}}=\limsup _{n \rightarrow \infty}\left|\sum_{k=1}^{n} a_{n k} X_{k}\right| \leq \sqrt{2 \rho} \text { a.s. }
$$

So, Theorem 1.4 in Chen and Gan [21] is a corollary of Theorem 8.

Remark 11. When $\alpha=1 / 2$, Theorem 8 also holds, but the proof is completely different. So, we will discuss it in the other paper.

\section{Proofs of the Main Results}

The main idea in the proofs of the main results is to use the following invariance principle (see Sakhanenko [22-24]), which is a powerful tool in the field of limit theory (e.g., see Csörgö et al. [25], Jiang and Zhang [26], Chen and Gan [21], and Chen and Wang [27]).

Lemma 12. Let $\left\{\xi_{k}, 1 \leq k \leq n\right\}$ be a sequence of independent random variables with $E \xi_{k}=0$ and $E \xi_{k}^{2}<\infty$ for $1 \leq k \leq n$. 
Then, there exists a sequence of independent normal random variables $\left\{\eta_{k}, 1 \leq k \leq n\right\}$ with $E \eta_{k}=0$ and $E \eta_{k}^{2}=E \xi_{k}^{2}$ such that, for all $q>2$ and $y>0$,

$$
P\left\{\left|\sum_{k=1}^{n} \xi_{k}-\sum_{k=1}^{n} \eta_{k}\right|>y\right\} \leq B y^{-q} \sum_{k=1}^{n} E\left|\xi_{k}\right|^{q},
$$

where $B$ is a positive constant depending only on $q$.

Proof of Theorem 1. For $1 \leq k \leq n$ and $n \geq 1$, we let

$$
\begin{aligned}
& Y_{n k}=X_{k} I\left(\left|X_{k}\right|>g(n)\right)-E X_{k} I\left(\left|X_{k}\right|>g(n)\right), \\
& Z_{n k}=X_{k}-Y_{n k} .
\end{aligned}
$$

We first prove that

$$
\sum_{n=1}^{\infty} n^{r-1} P\left\{\left|\sum_{k=1}^{n} a_{n k} X_{k}\right|>\varepsilon\right\}<\infty, \quad \forall \varepsilon>\sqrt{2 r \rho} .
$$

For $\varepsilon>\sqrt{2 r \rho}$, let $\varepsilon=\varepsilon_{1}+\varepsilon_{2}$, where $\varepsilon_{1}>0$ and $\varepsilon_{2}>\sqrt{2 r \rho}$. Then

$$
\begin{aligned}
& \sum_{n=1}^{\infty} n^{r-1} P\left\{\left|\sum_{k=1}^{n} a_{n k} X_{k}\right|>\varepsilon\right\} \\
& \leq \sum_{n=1}^{\infty} n^{r-1} P\left\{\left|\sum_{k=1}^{n} a_{n k} Y_{n k}\right|>\varepsilon_{1}\right\} \\
& \quad+\sum_{n=1}^{\infty} n^{r-1} P\left\{\left|\sum_{k=1}^{n} a_{n k} Z_{n k}\right|>\varepsilon_{2}\right\}:=I_{1}+I_{2} .
\end{aligned}
$$

For $I_{1}$, we have, by Markov's inequality, (12), (15), and a standard computation, that

$$
\begin{aligned}
I_{1} & \leq c \sum_{n=1}^{\infty} n^{r-1} \sum_{k=1}^{n} a_{n k}^{2} E X^{2} I(|X|>g(n)) \\
& \leq c \sum_{n=1}^{\infty} \frac{n^{r-1}}{\log n} E X^{2} I(|X|>g(n)) \leq c E \frac{h^{r}(|X|)|X|^{2}}{\log h(|X|)}<\infty .
\end{aligned}
$$

For $I_{2}$, we will use Lemma 12. By Lemma 12, there exists an array $\left\{\eta_{n k}, 1 \leq k \leq n, n \geq 1\right\}$ of rowwise independent normal random variables with $E \eta_{n k}=0, E \eta_{n k}^{2}=E\left|a_{n k} Z_{n k}\right|^{2}$ such that, for all $q>2$ and $y>0$,

$$
\begin{aligned}
& P\left\{\left|\sum_{k=1}^{n} a_{n k} Z_{n k}-\sum_{k=1}^{n} \eta_{n k}\right|>y\right\} \\
& \leq B y^{-q} \sum_{k=1}^{n}\left|a_{n k}\right|^{q} E|X|^{q} I(|X| \leq g(n)) .
\end{aligned}
$$

For $\varepsilon_{2}>\sqrt{2 r \rho}$, let $\varepsilon_{2}=\varepsilon_{3}+\varepsilon_{4}$, where $\varepsilon_{3}>0$ and $\varepsilon_{4}>\sqrt{2 r \rho}$. Then

$$
\begin{aligned}
I_{2} \leq & \sum_{n=1}^{\infty} n^{r-1} P\left\{\left|\sum_{k=1}^{n} a_{n k} Z_{n k}-\sum_{k=1}^{n} \eta_{n k}\right|>\varepsilon_{3}\right\} \\
& +\sum_{n=1}^{\infty} n^{r-1} P\left\{\left|\sum_{k=1}^{n} \eta_{n k}\right|>\varepsilon_{4}\right\} \\
:= & I_{21}+I_{22} .
\end{aligned}
$$

Take $q>2$ such that $r-1-\alpha(q-2)<-1$. Then, we have, by (36), (11), (12), (16), and a standard computation, that

$$
\begin{aligned}
I_{21} & \leq c \sum_{n=1}^{\infty} n^{r-1} \sum_{k=1}^{n}\left|a_{n k}\right|^{q} E|X|^{q} I(|X| \leq g(n)) \\
& \leq c \sum_{n=1}^{\infty} \frac{n^{r-1} g^{-(q-2)}(n)}{\log n} E|X|^{q} I(|X| \leq g(n)) \\
& \leq c E \frac{h^{r}(|X|)|X|^{2}}{\log h(|X|)}<\infty .
\end{aligned}
$$

We finally prove that $I_{22}<\infty$. To do this, let $\delta>1$ be sufficiently close to 1 such that $\varepsilon_{4}>\delta \sqrt{2 r \rho}$. Then $\sum_{k=1}^{n} a_{n k}^{2} \leq \varepsilon_{4}^{2} /(2 r \delta \log n)$ for all large enough $n$. Let $N$ be a standard normal random variable. It is well known that $P\{|N|>x\} \sim \sqrt{2 / \pi} x^{-1} e^{-x^{2} / 2}$. Since $E X^{2}=1, E Z_{n k}^{2}=$ $E\{X I(|X| \leq g(n))-E X I(|X| \leq g(n))\}^{2} \leq 1$ for all $1 \leq k \leq n$ and $n \geq 1$. Hence, for all large enough $n$,

$$
\begin{aligned}
& P\left\{\left|\sum_{k=1}^{n} \eta_{n k}\right|>\varepsilon_{4}\right\} \leq P\left\{|N|>\frac{\varepsilon_{4}}{\sqrt{\sum_{k=1}^{n} a_{n k}^{2}}}\right\} \\
& \leq c \sqrt{\sum_{k=1}^{n} a_{n k}^{2} \exp \left\{-\frac{\varepsilon_{4}^{2}}{2 \sum_{k=1}^{n} a_{n k}^{2}}\right\}} \\
& \leq c(\log n)^{-1 / 2} n^{-r \delta},
\end{aligned}
$$

which gives $I_{22}<\infty$.

Next, we prove that

$$
\sum_{n=1}^{\infty} n^{r-1} P\left\{\left|\sum_{k=1}^{n} a_{n k} X_{k}\right|>\varepsilon\right\}=\infty, \quad \forall 0<\varepsilon<\sqrt{2 r \rho} .
$$


For $0<\varepsilon<\sqrt{2 r \rho}$, let $\varepsilon=-\varepsilon_{5}-\varepsilon_{6}+\varepsilon_{7}$, where $\varepsilon_{5}>0, \varepsilon_{6}>0$ and $0<\varepsilon_{7}<\sqrt{2 r \rho}$. Then

$$
\begin{aligned}
& \sum_{n=1}^{\infty} n^{r-1} P\left\{\left|\sum_{k=1}^{n} \eta_{n k}\right|>\varepsilon_{7}\right\} \\
& \leq \sum_{n=1}^{\infty} n^{r-1} P\left\{\left|\sum_{k=1}^{n} a_{n i} Y_{n k}\right|>\varepsilon_{5}\right\} \\
& +\sum_{n=1}^{\infty} n^{r-1} P\left\{\left|\sum_{k=1}^{n} a_{n k} Z_{n k}-\sum_{k=1}^{n} \eta_{n k}\right|>\varepsilon_{6}\right\} \\
& +\sum_{n=1}^{\infty} n^{r-1} P\left\{\left|\sum_{k=1}^{n} a_{n k} X_{k}\right|>\varepsilon\right\} .
\end{aligned}
$$

The first series on the right hand side converges by $I_{1}<\infty$. The second series converges by $I_{21}<\infty$. But by using $P\{|N|>$ $x\} \sim \sqrt{2 / \pi} x^{-1} e^{-x^{2} / 2}$, it is easy to show that the series on the left-hand side diverges. Hence, the last series on the righthand side also diverges. That is, (40) holds.

Proof of Theorem 8. Let $\delta>0$ be given. Let

$$
X_{n k}^{\prime}=X_{k} I\left(\left|a_{n k} X_{k}\right| \leq n^{-\Delta}\right)
$$

for $\Delta>0$ to be specified below and let

$$
T_{n}^{\prime}=\sum_{k=1}^{n} a_{n k} X_{n k}^{\prime}
$$

Let

$$
X_{n k}^{\prime \prime}=X_{k} I\left(\left|a_{n k} X_{k}\right|>\frac{\delta}{N}\right)
$$

for an integer $N$ to be specified below and let

$$
T_{n}^{\prime \prime}=\sum_{k=1}^{n} a_{n k} X_{n k}^{\prime \prime}
$$

Let

$$
X_{n k}^{\prime \prime \prime}=X_{k}-X_{n k}^{\prime}-X_{n k}^{\prime \prime}=X_{k} I\left(n^{-\Delta}<\left|a_{n k} X_{k}\right| \leq \frac{\delta}{N}\right)
$$

and let

$$
T_{n}^{\prime \prime \prime}=\sum_{k=1}^{n} a_{n k} X_{n k}^{\prime \prime \prime}
$$

Since $\delta>0$ is arbitrary, to prove (22), it is enough to show that

$$
\begin{gathered}
\limsup _{n \rightarrow \infty}\left|T_{n}^{\prime}-E T_{n}^{\prime}\right| \leq \sqrt{2 \rho} \text { a.s. } \\
E T_{n}^{\prime} \longrightarrow 0 \\
T_{n}^{\prime \prime} \longrightarrow 0 \text { a.s. } \\
\limsup _{n \rightarrow \infty}\left|T_{n}^{\prime \prime \prime}\right| \leq \delta \text { a.s. }
\end{gathered}
$$

Using the same argument as in the proof of Theorem 1, we have that, for any $\varepsilon>\sqrt{2 \rho}$ with $\varepsilon=\varepsilon_{1}+\varepsilon_{2}$, where $\varepsilon_{1}>\sqrt{2 \rho}$ and $\varepsilon_{2}>0$,

$$
\begin{aligned}
& \sum_{n=1}^{\infty} P\left\{\left|T_{n}^{\prime}-E T_{n}^{\prime}\right|>\varepsilon\right\} \\
& \leq \sum_{n=1}^{\infty}\left(P\left\{\left|\sum_{k=1}^{k} \eta_{n k}\right|>\varepsilon_{1}\right\}\right. \\
& \left.+P\left\{\left|T_{n}^{\prime}-E T_{n}^{\prime}-\sum_{k=1}^{k} \eta_{n k}\right|>\varepsilon_{2}\right\}\right) \\
& \leq c \sum_{n=1}^{\infty} \sqrt{\sum_{k=1}^{n} a_{n k}^{2} \exp \left\{-\frac{\varepsilon_{1}^{2}}{2 \sum_{k=1}^{n} a_{n k}^{2}}\right\}} \\
& +c \sum_{n=1}^{\infty} \sum_{k=1}^{n} E\left|a_{n k} X_{n k}^{\prime}\right|^{q} \\
& \leq c \sum_{n=1}^{\infty} \exp \left\{-\frac{\varepsilon_{1}^{2}}{2 \sum_{k=1}^{n} a_{n k}^{2}}\right\} \\
& \quad+c \sum_{n=1}^{\infty} n^{1-q \Delta}<\infty
\end{aligned}
$$

when $q>2$ is large enough. Hence, by Borel-Cantelli lemma, $\lim \sup _{n \rightarrow \infty}\left|T_{n}^{\prime}-E T_{n}^{\prime}\right| \leq \sqrt{2 \rho}$ a.s. Taking $r \in(2,1 / \alpha)$, we get, by $E X=0$, (11), and (22), that

$$
\begin{aligned}
\left|E T_{n}^{\prime}\right| & \leq \sum_{k=1}^{n} E\left|a_{n k} X_{k}\right| I\left(\left|a_{n k} X_{k}\right|>n^{-\Delta}\right) \\
& \leq n^{(r-1) \Delta} \sum_{k=1}^{n} E\left|a_{n k} X_{k}\right|^{r} I\left(\left|a_{n k} X_{k}\right|>n^{-\Delta}\right) \\
& \leq c n^{(r-1) \Delta} \sum_{k=1}^{n}\left|a_{n k}\right|^{r} \leq c n^{(r-1) \Delta}(g(n))^{-(r-2)} ;
\end{aligned}
$$

Hence, $E T_{n}^{\prime} \rightarrow 0$ when $\Delta$ is small enough. By $E h(|X|)<\infty$,

$$
\sum_{k=1}^{\infty} P\left\{|X|>\left(\frac{\delta}{N K}\right) g(k)\right\}<\infty
$$

which implies that the series $\sum_{k=1}^{\infty}\left|X_{k}\right| I\left(\left|X_{k}\right|>(\delta / N K) g(k)\right)$ converges almost surely by Borel-Cantelli lemma. Hence,

$$
\begin{aligned}
\left|T_{n}^{\prime \prime}\right| & \leq K g^{-1}(n) \sum_{k=1}^{n}\left|X_{k}\right| I\left(\left|X_{k}\right|>\left(\frac{\delta}{N K}\right) g(n)\right) \\
& \leq K g^{-1}(n) \sum_{k=1}^{\infty}\left|X_{k}\right| I\left(\left|X_{k}\right|>\left(\frac{\delta}{N K}\right) g(k)\right) \longrightarrow 0 \text { a.s. }
\end{aligned}
$$

To prove $\lim \sup _{n \rightarrow \infty}\left|T_{n}^{\prime \prime \prime}\right| \leq \delta$ a.s., by Borel-Cantelli lemma, it is enough to show that

$$
\sum_{n=1}^{\infty} P\left\{\left|T_{n}^{\prime \prime \prime}\right|>\delta\right\}<\infty
$$


Since $X_{n k}^{\prime \prime \prime}=X_{k} I\left(n^{-\Delta}<\left|a_{n k} X_{k}\right| \leq \delta / N\right),\left|T_{n}^{\prime \prime \prime}\right|=$ $\left|\sum_{k=1}^{n} a_{n k} X_{n k}^{\prime \prime \prime}\right|>\delta$ implies that there must exist at least $N$ indices $k$ such that $X_{n k}^{\prime \prime \prime} \neq 0$. Hence, by Bonferroni's inequality (see, e.g., Lemma 4.1.2 in [1]) and Markov's inequality,

$$
\begin{aligned}
P\left\{\left|T_{n}^{\prime \prime \prime}\right|>\delta\right\} \leq & P\{\text { there exist at least } N \\
& \left.\quad \text { indices } k \text { such that } X_{n k}^{\prime \prime \prime} \neq 0\right\} \\
& \leq \sum_{1 \leq k_{1}<k_{2}<\cdots<k_{N} \leq n} \prod_{j=1}^{N} P\left\{\left|a_{n k_{j}} X_{k_{j}}\right|>n^{-\Delta}\right\} \\
& \leq\left(\sum_{k=1}^{n} P\left\{\left|a_{n k} X_{k}\right|>n^{-\Delta}\right\}\right)^{N} \\
& \leq\left(n^{p \Delta} \sum_{k=1}^{n}\left|a_{n k}\right|^{p} E|X|^{p}\right)^{N} \\
& \leq c n^{p \Delta N}(g(n))^{-N(p-2)}
\end{aligned}
$$

where $p \in(2,1 / \alpha)$. Thus, choosing $\Delta$ sufficiently small and $N$ sufficiently large such that $N[\alpha(p-2)-p \Delta]>1$, we have

$$
\sum_{n=1}^{\infty} P\left\{\left|T_{n}^{\prime \prime \prime}\right|>\delta\right\} \leq c \sum_{n=1}^{\infty} n^{p \Delta N}[g(n)]^{-N(p-2)}<\infty
$$

since the function $x^{p \Delta N}[g(x)]^{-N(p-2)}$ is regularly varying at infinity with index $-N[\alpha(p-2)-p \Delta]<-1$. This completes the proof.

\section{Conflict of Interests}

The authors declare that there is no conflict of interests regarding the publication of this paper.

\section{Acknowledgments}

The authors would like to thank the referees for the helpful comments. The research of Pingyan Chen is supported by the National Natural Science Foundation of China (11271161). The research of Soo Hak Sung is supported by Basic Science Research Program through the National Research Foundation of Korea (NRF) funded by the Ministry of Education, Science and Technology (2010-0013131).

\section{References}

[1] W. F. Stout, Almost Sure Convergence, Academic Press, New York, NY, USA, 1974.

[2] W. F. Stout, "Some results on the complete and almost sure convergence of linear combinations of independent random variables and martingale differences," Annals of Mathematical Statistics, vol. 39, pp. 1549-1562, 1968.

[3] P. L. Hsu and H. Robbins, "Complete convergence and the law of large numbers," Proceedings of the National Academy of Sciences of the United States of America, vol. 33, pp. 25-31, 1947.
[4] S. H. Sung, "Complete convergence for weighted sums of arrays of rowwise independent $B$-valued random variables," Stochastic Analysis and Applications, vol. 15, no. 2, pp. 255-267, 1997.

[5] F. Y. Cheng and Y. B. Wang, "Complete convergence for weighted sums of arrays of $B$-valued random variables," Journal of Mathematical Research and Exposition, vol. 24, no. 4, pp. 665669, 2004.

[6] Q. Wu, "Complete convergence for negatively dependent sequences of random variables," Journal of Inequalities and Applications, vol. 2010, Article ID 507293, 10 pages, 2010.

[7] S. H. Sung, "Complete convergence for weighted sums of negatively dependent random variables," Statistical Papers, vol. 53, no. 1, pp. 73-82, 2012.

[8] M. L. Katz, "The probability in the tail of a distribution," Annals of Mathematical Statistics, vol. 34, pp. 312-318, 1963.

[9] L. E. Baum and M. Katz, "Convergence rates in the law of large numbers," Transactions of the American Mathematical Society, vol. 120, pp. 108-123, 1965.

[10] T. L. Lai, "Limit theorems for delayed sums," Annals of Probability, vol. 2, pp. 432-440, 1974.

[11] D. L. Li, M. B. Rao, T. F. Jiang, and X. C. Wang, "Complete convergence and almost sure convergence of weighted sums of random variables," Journal of Theoretical Probability, vol. 8, no. 1, pp. 49-76, 1995.

[12] B.-Y. Jing and H.-Y. Liang, "Strong limit theorems for weighted sums of negatively associated random variables," Journal of Theoretical Probability, vol. 21, no. 4, pp. 890-909, 2008.

[13] K. Budsaba, P. Chen, K. Panishkan, and A. Volodin, "Strong laws for certain types of $U$-statistics based on negatively associated random variables," Siberian Advances in Mathematics, vol. 19, no. 4, pp. 225-232, 2009.

[14] L. V. Thanh and G. Yin, "Almost sure and complete convergence of randomly weighted sums of independent random elements in Banach spaces," Taiwanese Journal of Mathematics, vol. 15, no. 4, pp. 1759-1781, 2011.

[15] N. H. Bingham, C. M. Goldie, and J. L. Teugels, Regular Variation, vol. 27, Cambridge University Press, Cambridge, UK, 1987.

[16] T. C. Hu and N. C. Weber, "On the rate of convergence in the strong law of large numbers for arrays," Bulletin of the Australian Mathematical Society, vol. 45, no. 3, pp. 479-482, 1992.

[17] Y. C. Qi, “On strong convergence of arrays," Bulletin of the Australian Mathematical Society, vol. 50, no. 2, pp. 219-223, 1994.

[18] D. L. Li, M. B. Rao, and R. J. Tomkins, "A strong law for B-valued arrays," Proceedings of the American Mathematical Society, vol. 123, no. 10, pp. 3205-3212, 1995.

[19] P. Embrechts and M. Maejima, "The central limit theorem for summability methods of i.i.d. random variables," Zeitschrift für Wahrscheinlichkeitstheorie und Verwandte Gebiete, vol. 68, no. 2, pp. 191-204, 1984.

[20] T. L. Lai, "Summability methods for independent identically distributed random variables," Proceedings of the American Mathematical Society, vol. 45, pp. 253-261, 1974.

[21] P. Chen and S. Gan, "Limiting behavior of weighted sums of i.i.d. random variables," Statistics \& Probability Letters, vol. 77, no. 16, pp. 1589-1599, 2007.

[22] A. I. Sakhanenko, "On unimprovable estimates of the rate of convergence in the invariance principle," in Colloquia Mathematica Societatis János Bolyai, pp. 779-783, Nonparametric Statistical Inference, Budapest, Hungary, 1980. 
[23] A. I. Sakhanenko, "On estimates of the rate of convergence in the invariance principle," in Advances in Probability Theory: Limit Theorems and Related Problems, A. A. Borovkov, Ed., pp. 124-135, Springer, New York, NY, USA, 1984.

[24] A. I. Sakhanenko, "Convergence rate in the invariance principle for nonidentically distributed variables with exponential moments," in Advances in Probability Theory: Limit Theorems for Sums of Random Variables, A. A. Borovkor, Ed., pp. 2-73, Springer, New York, NY, USA, 1985.

[25] M. Csörgö, B. Szyszkowicz, and Q. Wang, "Donsker's theorem for self-normalized partial sums processes," The Annals of Probability, vol. 31, no. 3, pp. 1228-1240, 2003.

[26] Y. Jiang and L. X. Zhang, "Precise rates in the law of iterated logarithm for the moment of i.i.d. random variables," Acta Mathematica Sinica, vol. 22, no. 3, pp. 781-792, 2006.

[27] P. Y. Chen and D. C. Wang, "Convergence rates for probabilities of moderate deviations for moving average processes," Acta Mathematica Sinica, vol. 24, no. 4, pp. 611-622, 2008. 


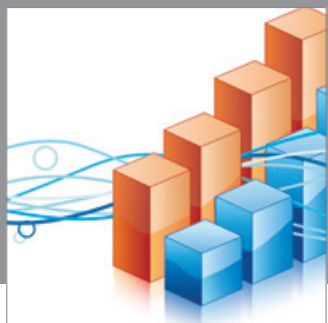

Advances in

Operations Research

mansans

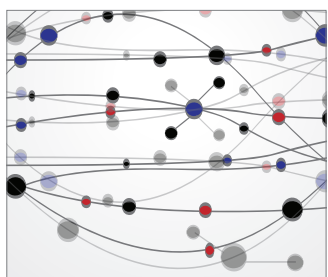

The Scientific World Journal
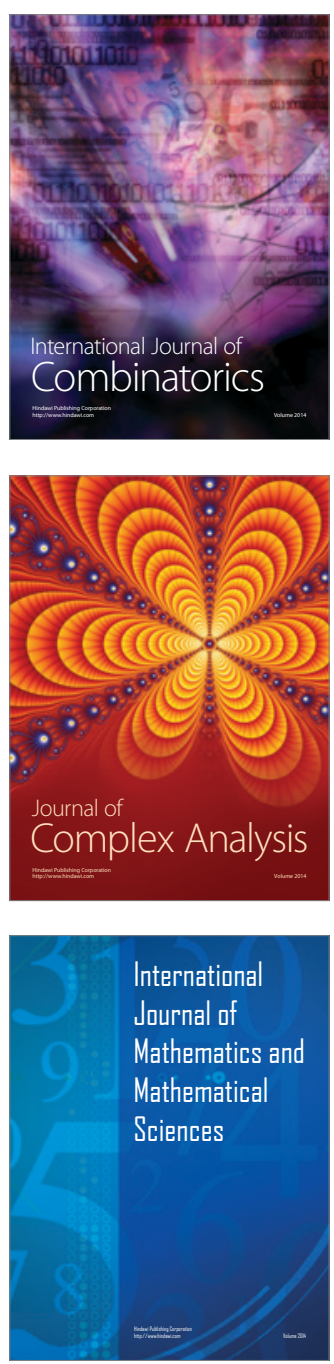
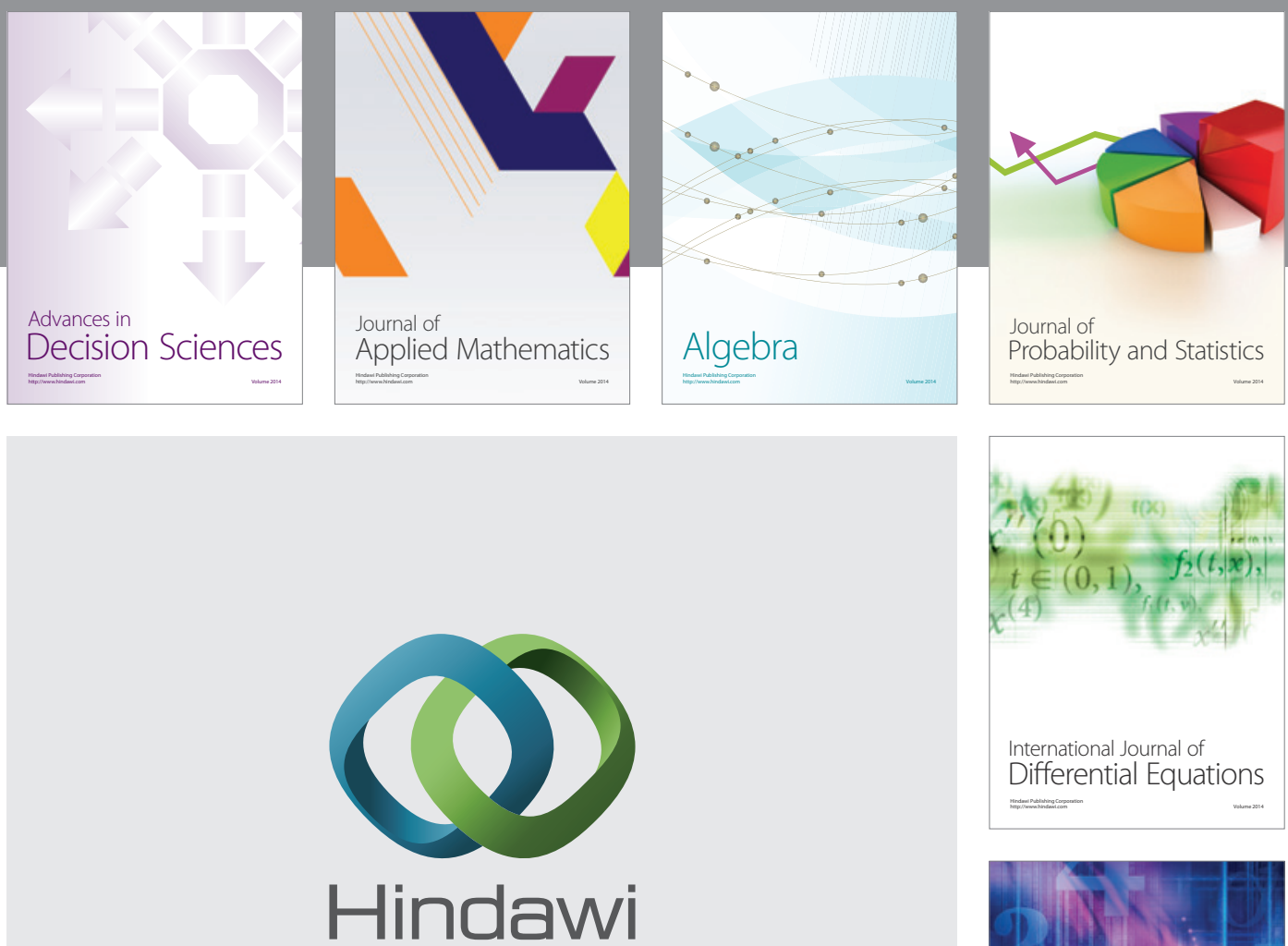

Submit your manuscripts at http://www.hindawi.com
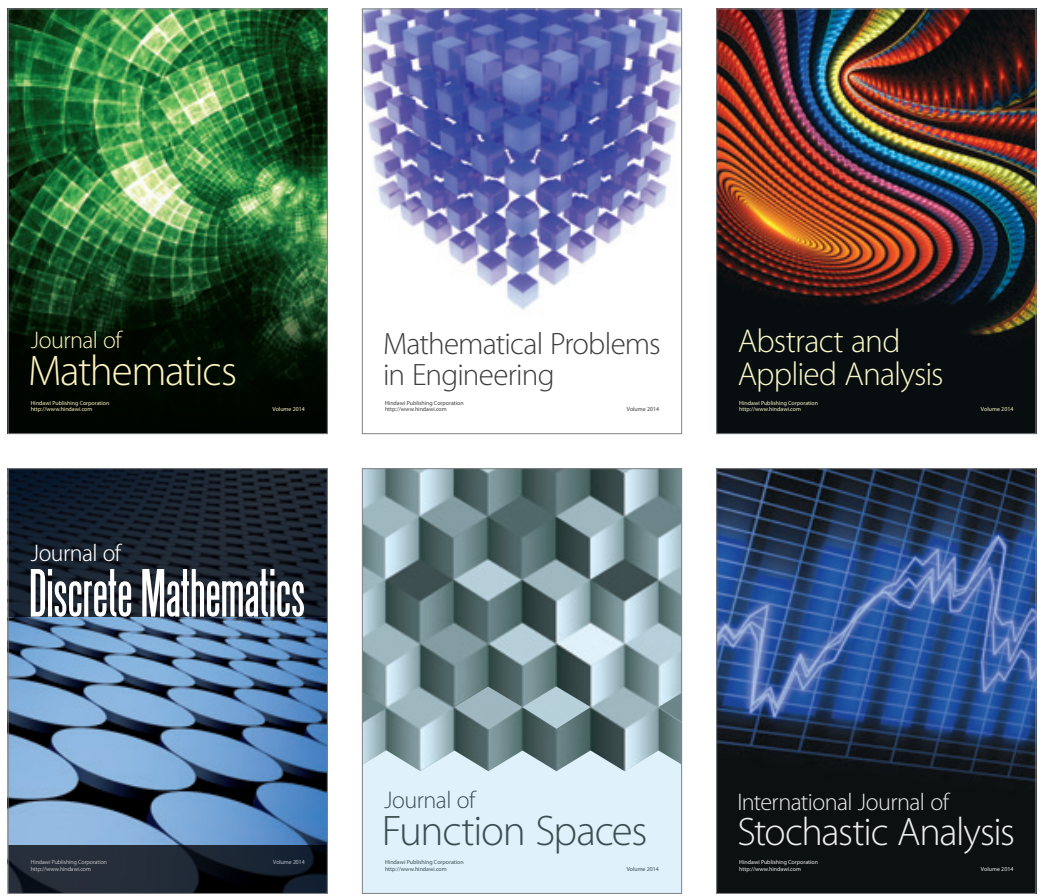

Journal of

Function Spaces

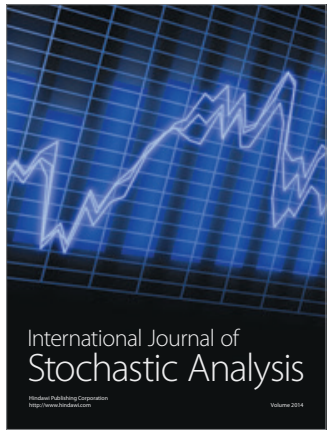

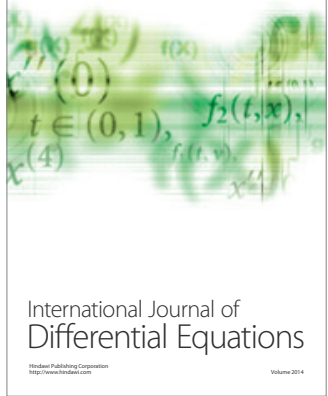
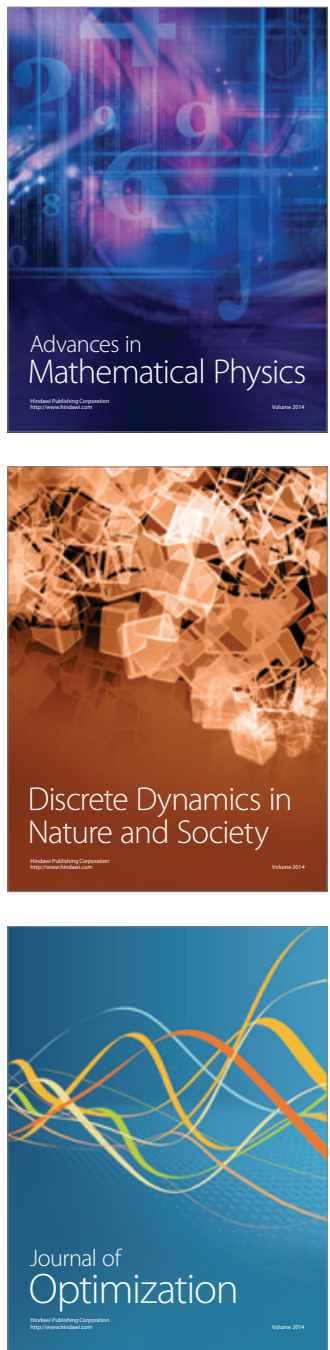